\title{
Effect of Laser Fluence on the Characteristics of Ag Nanoparticles Produced by Laser Ablation
}

\author{
Davoud Dorranian*, Shiva Tajmir, Farzane Khazanehfar \\ Laser Lab., Plasma Physics Research Center, Science and Research Branch, Islamic Azad University, Tehran, Iran. \\ Email: *doran@srbiau.ac.ir
}

Received August $12^{\text {th }}, 2013$; revised September 12 $2^{\text {th }}, 2013$; accepted September $20^{\text {th }}, 2013$

Copyright (C) 2013 Davoud Dorranian et al. This is an open access article distributed under the Creative Commons Attribution License, which permits unrestricted use, distribution, and reproduction in any medium, provided the original work is properly cited.

\begin{abstract}
$6-12 \mathrm{~nm}$ silver nanoparticles were synthesized by the pulsed laser ablation of a high purity silver bulk in distilled water. Effect of laser fluence on the size, morphology and structure of produced nanoparticles has been studied experimentally. Pulses of a Q-switched Nd:YAG laser of 1064 wavelength at $7 \mathrm{~ns}$ pulse width at different fluences were employed to irradiate the silver target in water. The UV-Visible absorption spectra of the Ag nanoparticles exhibit surface plasmon resonance absorption peak in the UV region. TEM and SEM micrographs, in a good agreement with DLS results, indicate that with increasing the laser fluence the average size of spherical Ag nanoparticles is decreased. It is found that $\mathrm{Ag}$ nanoparticles exhibit photoluminescence emission with two peaks because of the inter band transition and electron-hole recombination.
\end{abstract}

Keywords: Laser Ablation; Nanoparticle; Absorption Spectra; XRD; TEM; SEM; Mie Theory; Surface Plasmon Resonance; DLS

\section{Introduction}

There is a growing interest in the fabrication of nanomaterials and their applications in various fields of life and technology such as electronics, health care, energy generation, and storage. Nanomaterials, defined as materials having typical dimensions less than $100 \mathrm{~nm}$, present very special physical and chemical properties that strongly depend on their size and shape [1]. Properties of material are known to be strongly dependent on the chemical nature and the structure of its constituents, in particular, due to overlapping their atomic or molecular orbitals. Bulk materials composed of a large number of atoms, are characterized by the presence of energy bands, which are responsible for most of physical and chemical properties of solids. However, for nanomaterials the number of atoms becomes so small that the electronic energy bands are significantly modified, strongly affecting almost all physical properties of the materials [2]. Those significant properties, such as chemical, electronic, mechanical, and optical properties, of nanoparticles obviously distinguish them from those of the corresponding "bulk" material.

Production of nanoparticles (NPs) in the liquid medium can be done in several ways; Methods of chemical,

${ }^{*}$ Corresponding author. electrical, and laser ablation [3-5]. Evidence shows that the latter method is superior to other methods. Indeed, laser ablation in liquids, which consists of the pulverization of a solid target in liquid ambience, gives a unique opportunity to solve the toxicity problems. In contrast to chemical nanofabrication methods, laser ablation can be performed in a clean, well-controlled environment, such as deionized water, giving rise to the production of ultrapure nanomaterials [3]. Furthermore, different structural and morphological characters of produced NPs can be controlled by laser fluence, spot size, wavelength, pulse width, and repetition rate of laser pulse.

Noble metal NPs have attracted much attention because of their unique size dependent optical properties, magnetic properties, catalytic properties and etc [3]. The size dependent properties of silver NPs make them suitable for many applications in various areas such as catalytic, optical and antibacterial applications [6,7]. Silver NPsare proved to be more effective as it has good antimicrobial efficacy against bacteria, viruses and other eukaryotic micro-organisms [8]. The current investigations support that use of silver ion or metallic silver as well as silver NPs can be exploited in medicine for burn treatment, dental materials, coating stainless steel materials, textile fabrics, water treatment, sunscreen lotions, etc. 
and posses low toxicity to human cells, high thermal stability and low volatility [9]. Engineered nanoparticles are used in biomedical applications such as antibacterial implants or catheters, modification of textiles, and refinement of polymers [10]. Silver NPs are also used in biosensors for glucose monitoring in diabetes patients and other medical health related target [11-23].

In this manuscript we have investigated the effect of laser fluence on the characteristics of silver NPs produced by laser ablation of bulk silver in distilled water. Effect of laser fluence on the size distribution as well as lattice structure of NPs is studied experimentally and morphology of NPs is observed.

The manuscript is organized as follow; following the introduction in Section 1, the experimental setup is presented in Sections 2. 3 is devoted to results and discussions and Section 4 includes conclusion.

\section{Experimental Set up}

Nano particles were prepared by ablation of a high purity silver bulk in distilled water, using the fundamental harmonic of Nd:YAG laser operating at $1064 \mathrm{~nm}$ with pulse width of $7 \mathrm{~ns}$ and $10 \mathrm{~Hz}$ repetition rate. Silver bulk was placed at the bottom of a water contain with its surface at the focal point of a $80 \mathrm{~mm}$ convex lens. Height of water on the silver target was $12 \mathrm{~mm}$. Laser beam diameter was $6 \mathrm{~mm}$ before lens and was calculated to be $30 \mu \mathrm{m}$ on the surface of the target. The volume of the water in the ablation contain was $20 \mathrm{ml}$ and silver target was ablated with 500 laser pulses at different fluences. Samples 1 - 5 were prepared with laser pulse fluences of $1,1.5,2,2.5$, $3 \mathrm{~J} / \mathrm{cm}^{2}$ respectively.

Optical absorption spectra of samples in a $10 \mathrm{~mm}$ path length quartz cells were measured by UV-Vis-NIR spectrophotometer from PG Instruments (T-80). Dynamic Light Scattering (DLS) measurement was done using the Nano ZS (red badge) ZEN 3600 device from Malvern Co, for studying the hydrodynamic size distribution of the nanoparticles in water. Normally 100 or more particles were counted to determine the size distribution of each sample. TEM and SEM micrographs were taken usingCM120 and XL30 systems form PHILIPS Co respectively. X-ray diffraction was measured, employing $\mathrm{X}$ PERTPRO X-ray diffract-meter from the PAN alitical.

To measure the ablation rate in the liquid medium, the target was weighed up before and after the ablation process by Sartorius Utensil model CP225D with 0.01 $\mathrm{mg}$ readability. After ablation inside water with 500 laser pulses, the target was dried and weighed up again. Hence, the mass loss of the target attributed to the amount of generated NPs.

\section{Results and Discussion}

In Figure 1 the photo of samples are presented and in
Figure 2 the ablation rate of samples versus laser fluence is plotted. Depending on nanoparticle size and concentration, the color of silver nanoparticle solution in water can be varied from yellow to brown as is shown in Figure 1. The color of sample 1 is light yellow which changes gradually to brown in sample 5 . One reason can be due to amount of nano particles in the solutions. In Figure 2 it is shown that the amount of silver nanoparticles is increased exponentially with increasing the laser pulse fluence. Another reason for changing the color of samples is due to their size. Measurements show that the size of nanoparticles is decreased from s1 to s5. With decreasing the size of NPs their color is changed from yellow to brown.

The XRD pattern of dried powder of generated NPs is shown in Figure 3. Diffraction peaks are clearly observed and are located in positions consistent with those expected for Ag, as indicated by the Joint Committee on Powder Diffraction Standards (JCPDS) plot presented beneath the experimental data. The preferred orientation of Ag bulk target and generated NP samples are exactly the same. All have a poly crystal structure with several preferred orientations. Therefore, it can be claimed that in

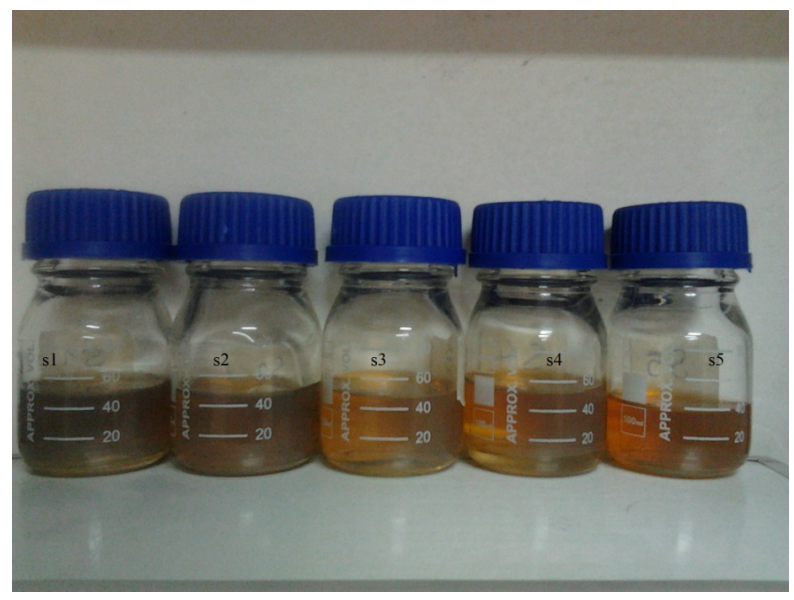

Figure 1. Ag nanoparticles samples in distilled water.

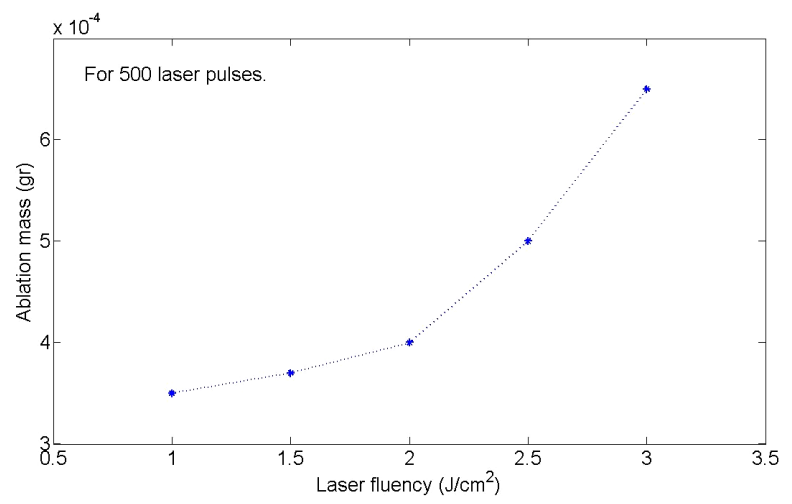

Figure 2. Variation of the mass of the target during the ablation versus laser pulse fluency. 


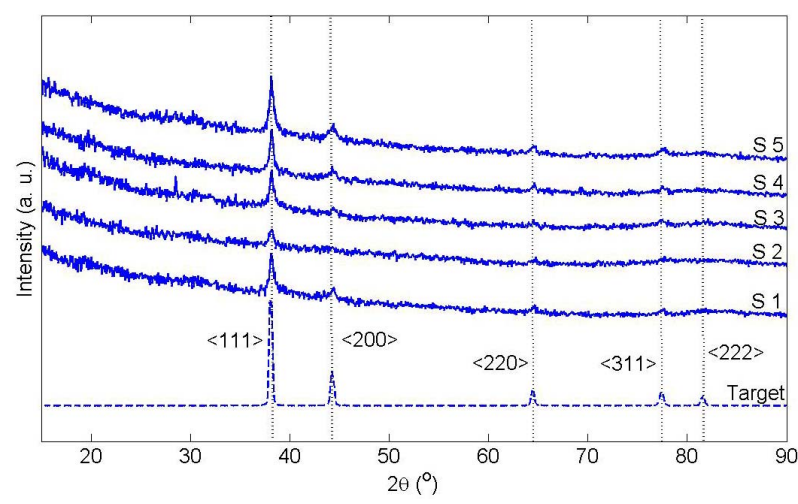

Figure 3. X-ray diffraction pattern of Ag target and Ag nanoparticle samples after drying.

this regime of ablation Ag nanoparticles do not produced by nucleation of Ag atoms. NPs are ablated from the bulk target with the same lattice structure. The mean grain size of synthesized Ag nano particles could be estimated according to the Debye-Scherrermodel. Broadening the peaks of XRD pattern of NPs from s1 to s5 confirm that size of grains is decreased in these samples [8].

Absorption spectrums of nanoparticle solutions are presented in Figure 4. The absorption peaks at $405 \mathrm{~nm}$ are due to silver NPs Surface Plasmon Resonances (SPR). For SPR phenomenon to happen, the particle must be much smaller than the wavelength of incident light. The intensity of absorption peak depends on the number of nanoparticles. For different samples this number is different in the experiment. If the size of NPs increases, their SPR absorption spectrum peak will be shifted toward larger wavelength and vise-versa [9]. From s1 to s5 a small blue shift of $5 \mathrm{~nm}$ can be observed for the SPR peaks of absorption spectrum. Another important parameter of SPR peak is its Full Width at Half Maximum (FWHM). It is shown in Mie scattering theory that with increasing the size of NPs, FWHM of their SPR peak will decrease. In the case of produced NPs here, the FWHMs of SPR peaks are very close of about $88 \mathrm{~nm}$. It is due to the sizes of produced NPs which are close and the fact that FWHM of SPR peak is not as sensitive to NPs size as the wavelength of the SPR.

Figures 5 present SEM images of Ag nanostructures prepared by laser ablation in deionized water. Images are prepared with $25 \mathrm{kV}$ electrons leads to $30 \mathrm{kX}$ magnification. Nps solutions were dried on $\mathrm{Al}$ foil at room temperature before imaging. Morphology of Ag nanoparticles depends on laser pulse energy. The generated grains of nanoparticles in this experimental condition are almost spherical and adhered to each other. This structure is similar to those reported in Ref. 11 prepared at room temperature. It is observable that with increasing the energy of laser pulse, rate of adhesion is decreased and grains are smaller. Specially in the case of s5 there is not

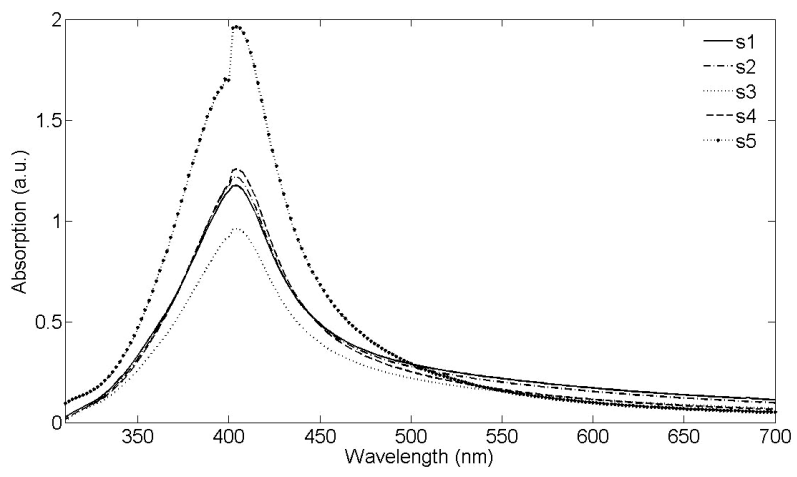

Figure 4. UV-Vis-NIR absorption spectrum of Ag nanoparticles in distilled water, with distilled water as the reference.

any grain forms in the nanoparticle solution. The size of $\mathrm{Ag}$ nanoparticles is decreased with increasing the fluence of the laser pulse.

More information about the size and morphology of NPs are presented in Figures 6 and 7(a)-(e). In Figure 6 the hydrodynamic size distribution of samples measured by DLS is shown. Hydrodynamic size of nanoparticles is their real diameter plus the diameter of the electrostatic potential around them, so it is larger than the real size of NPs. The peaks of hydrodynamic size distribution functions of samples 1 , and 2 have one peak at $78.8 \mathrm{~nm}$. The peak of sample 3 is occurred at $68.1 \mathrm{~nm}$. Hydrodynamic size distribution functions of samples 4 and 5 have two peaks. The peak occurred at larger size is due to ablated NPs while the peak corresponds to smaller size is due to ablated NPs which are fragment by the laser pulse energy. The second illumination process has extenuated the ablated nanoparticles. In the case of lower laser pulse energy, this process can not be observed. For sample 4, peaks are occurred at $58.8 \mathrm{~nm}$ and $24.4 \mathrm{~nm}$ while for sample 5 peaks are occurred at $10.1 \mathrm{~nm}$ and $3.1 \mathrm{~nm}$. With increasing the laser fluence in this regime of ablation, the size of NPs is decreased. For laser pulse energy density of $2.5 \mathrm{~J} / \mathrm{cm}^{2}$ and $3 \mathrm{~J} / \mathrm{cm}^{2}$ the secondary irradiation of NPs by the laser pulse has generated another class of smaller Ag nanoparticles.

TEM images of samples are presented in Figures 7 (a)-(e). In this set of images, the in tergrain structure can be observed. Produced nanoparticles are spherical without any aggregation. The size distribution of nanoparticles can also be observed in Figure 7. These graphs are plotted using the "measurement" software. We have a wide range of sizes of nanoparticles in each sample, but from sample 1 to 5 we can see that the peak of size distribution of samples is tended to smaller values from 12 $\mathrm{nm}$ to $6 \mathrm{~nm}$.

Considering that TEM and DLS analyses particle sizes differently, the results obtained from DLS and TEM can be considered to be in good agreement with each other. In both cases with increasing the laser fluence, the size of 


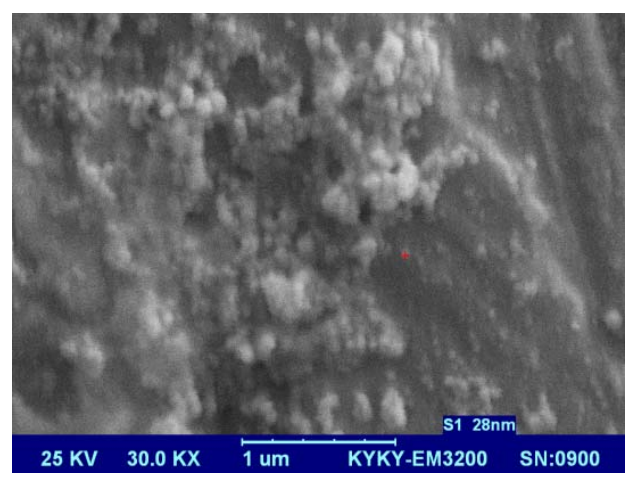

s 1



s3

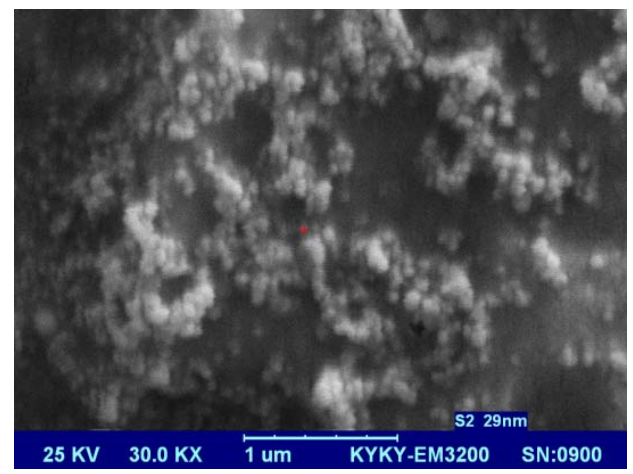

s2

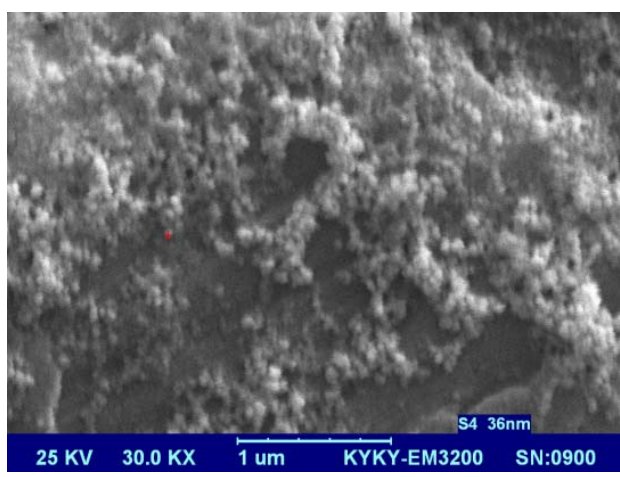

s4

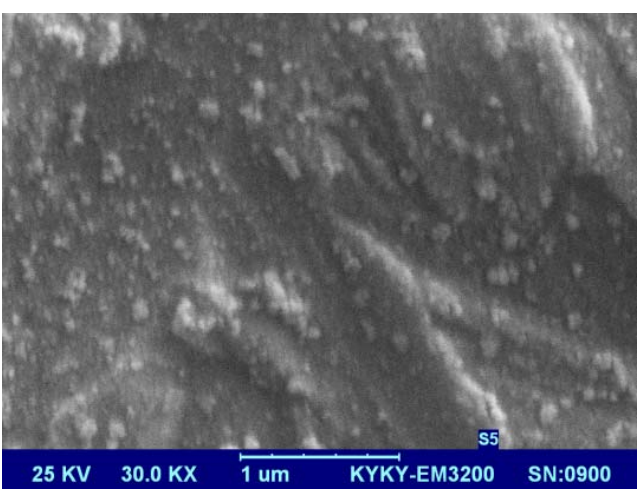

s5

Figure 5. SEM micrographs of Ag nanoparticle samples dried on aluminum foil.

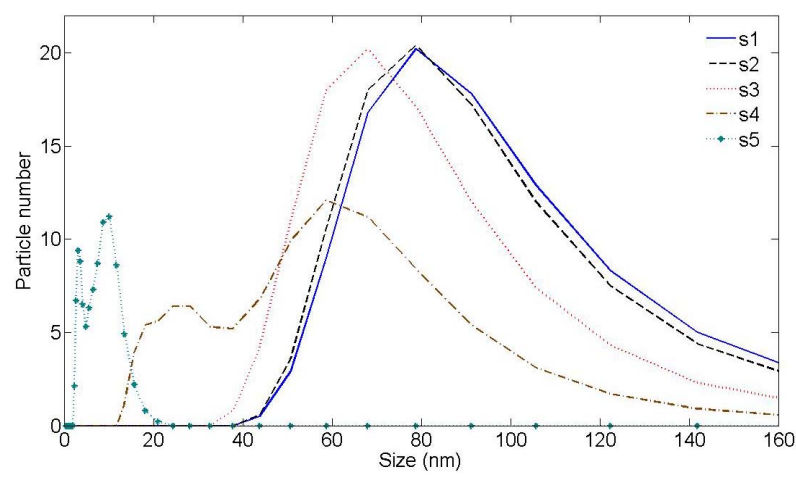

Figure 6. Ag nanoparticle hydrodynamic size distribution of samples measured by Dynamic Light Scattering (DLS) device.
NPs is decreased. But measured size by DLS device is larger than the sizes we can observe in TEM micrographs. This difference can be due to the fact that DLS analysis includes the ligand shell and determines the hydrodynamic sizes of the synthesized nanoparticles whereas in TEM we can look at only metallic core $[11,23]$.

The photoluminescence spectra from Ag NPs are shown in Figure 8. Photoluminescence emissions spectra from the synthesized samples have been recorded at room temperature at $270 \mathrm{~nm}$ excitation wavelengths. As can be seen in Figure 8, the PL emission intensity has been increased with increasing the laser fluence. The intensity of peaks may be intensively influenced by the increase of interactions between NPs due to higher con- 

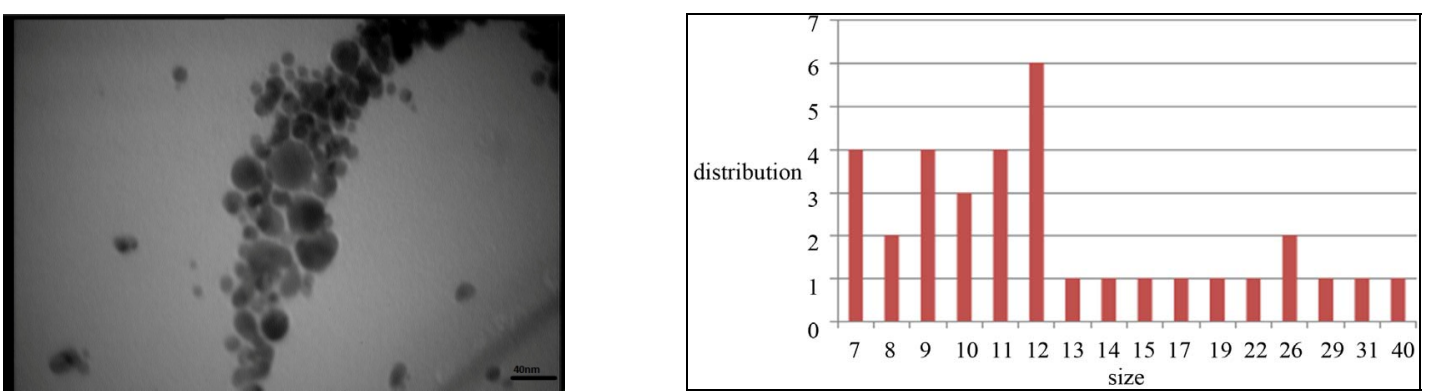

s1
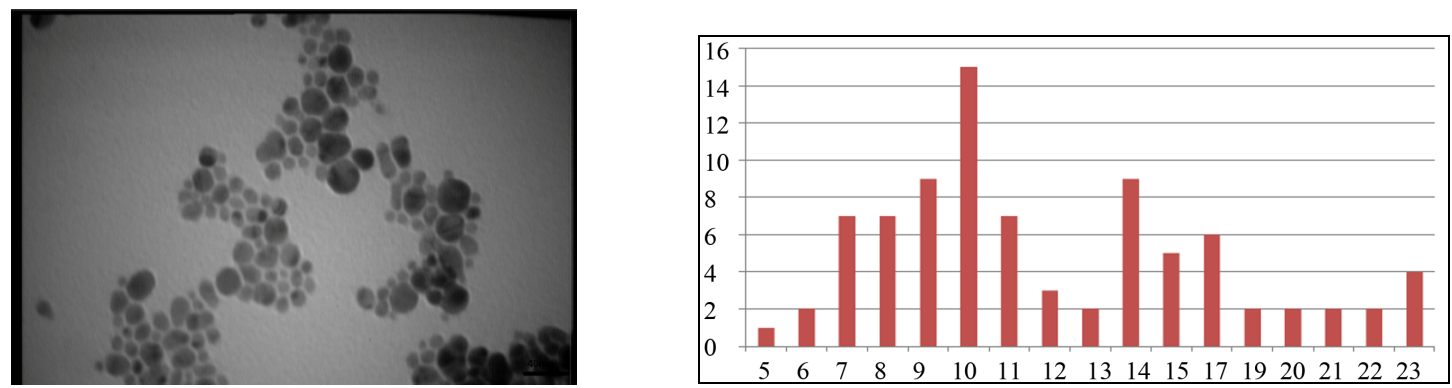

s2

s4


s5

Figure 7. TEM image and size distribution of Ag nanoparticle generated in distilled water with laser ablation mechanism. 
centration. It is found from Figure 8 that samples exhibit PL emissions in the violet-blue and UV region. There is one intense peak in the photoluminescence of all samples which is occurred in the range of 422 to $455 \mathrm{~nm}$. This peak shows a red shift from sample 1 to 5 respectively. There is another peak in the photoluminescence of samples in the range of $355-360 \mathrm{~nm}$. The intensity of this peak is increased noticeably from sample 1 to 5 and the peak is blue shifted. The schematic of the excitation and recombination mechanisms can be depicted as shown in Figure 9, where the band structure for a typical noble metal is represented by a simple model. The band corresponds to $355-360 \mathrm{~nm}$ transition is observed only in the spectra of the samples containing the smallest Ag NPs which are typically smaller than $10 \mathrm{~nm}$ and is absent in the samples with larger particles. This peak is very close to the peak of PL band of bulk silver [24-26]. This emission is corresponded to the direct radiative inter band recombination of the conduction sp-band electrons with holes in the valance $d$ band that have been scattered to momentum states less that the Fermi momentum. The red shift of this peak may be due to coupling of these photons with SPR photons. The band corresponds to 422 $455 \mathrm{~nm}$ is in the wavelength range of SPR excitation which is red shifted when the size of the particles de-



Figure 8. Photoluminescence spectra of Ag nanoparticles in distilled water.

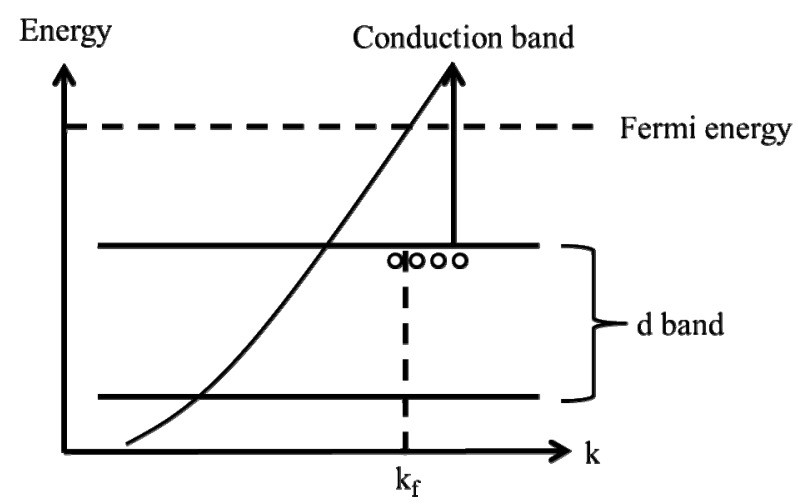

Figure 9. Excitation and recombination mechanism inside the band structure of noble metals. creases. This behavior can be attributed to radiative decay of SPR excited in silver NPs [27-29].

In Figure 6, DLS size measurement shows that we have two classes of NPs in the s4 and s5. In case of s1 s3 there is just one peak in the size distribution function measured by DLS method. We can see that the difference of energy of conduction band and d-band in the smaller NPs in s4 and s5 is larger than the similar magnitude in larger size NPs. It is natural, since in this case, the perturbation of energy levels in small NPs is smaller. A blue shift is taken place for this peak from s4 to s5.

\section{Conclusion}

Preparation of silver NPs by laser ablation method at different fluencies of laser pulse in pure water is investigated. The generated NPs in this experimental condition are almost spherical. The size of NPs is decreased by increasing the laser pulse fluency and the rate of adhesion is decreased and grains are smaller. The intensity of absorption peak depends on the laser pulse fluence. The poly crystal structure of generated NPs and the Ag target are exactly same. The room temperature photoluminescence spectra of silver nanoparticles show increasing the intensity of emission with increasing the laser pulse fluency. With increasing the laser fluence a smaller class of NPs is formed due to secondary interaction of laser pulse and ablated NPs. Effect of this smaller class is clear in DLS hydrodynamic size distribution and PL spectrums.

\section{REFERENCES}

[1] C. Baker, A. Pradhan, L. Pakstis, J. Pochan Darrin, Shah S. Ismat, "Synthesis and Antibacterial Properties of Silver Nanoparticles," Journal of Nanoscience and Nanotechnology, Vol. 5, No. 2, 2005, pp. 244-249.

[2] A. Pyatenko, K. Shimokawa, M. Yamaguchi, O. Nishimura and M. Suzuki, "Synthesis of Silver Nanoparticles by Laser Ablation in Pure Water," Applied Physics A, Vol. 79, No. 4-6, 2004, pp. 803-806. http://dx.doi.org/10.1007/s00339-004-2841-5

[3] P. Mulvaney, T. Linnert and A. Henglein, "Surface Chemistry of Colloidal Silver in Aqueous Solution: Observations on Chemisorption and Reactivity," The Journal of Physical Chemistry, Vol. 95, 1991, pp. 7843-7846.

[4] S. Gunasagaran, T. Zi Xin, T. Foo, W. Xiang, E. Fang, C. Yang, N. Jeyakaumar and S. Arumugam Dhanaraj, "Biosynthesis of Silver Nanoparticles Using Mangosteen Leaf Extract and Evaluation of Their Antimicrobial Activities," Journal Of Saudi Chemical Of Society, Vol. 15, No. 113, 2011, pp. 113-120.

[5] E. Solati, M. Mashayekh and D. Dorranian, "Effects of Laser Pulse Wavelength and Laser Fluenceon the Characteristics of Silver Nanoparticle Generated by Laserablation" Applied Physics A, Vol. 112, 2012, pp. 684-694.

[6] S. Lee, H. Jung, J. Shin and M. Chio, "Fabrication of 
Nano-Structured Hemispheres and Nanopillars Using Laterally Migrating Polymer Templates," Journal of Nanoscience and Nanotechnology, Vol. 56, No. 660, 2012, pp. 6008-6012.

[7] P. Gong, H. Li, X. He, K. Wang, J. Hu, S. Zhang and X. Yang, "Preparation and Antibacterial Activity of $\mathrm{Fe}_{3} \mathrm{O}_{4} \mathrm{Ag}$ Nanoparticles" Nanotechnology, Vol. 18, No. 28, 2007, Article ID: 285604.

[8] N. Durán, P. D. Marcarto, G. I. H. De Souza, O. L. Alves and E. Esposito, "Antibacterial Effect of Silver Nanoparticles Produced by Fungal Process on Textile Fabrics and Their Effluent Treatment," Journal of Biomedical Nanotechnology, Vol. 3, No. 2, 2007, pp. 203-208. http://dx.doi.org/10.1166/ibn.2007.022

[9] S. Barcikowski, A. Hahn, A. V. Kabashin and B. N. Chichkov, "Properties of Nanoparticles Generated during Femtosecond Laser Machining in Air and Water," Applied Physics, Vol. 87, No. 47, 2007, pp. 47-55.

[10] D. Dorranian, E. Solati, L. Dejam, "Photoluminescence of $\mathrm{ZnO}$ Nanoparticles Generated by Laser Ablation in Deionized Water," Applied Physics A, Vol. 109, No. 2, 2012, pp. 307-314.

http://dx.doi.org/10.1007/s00339-012-7073-5

[11] M. T. Lee, D. J. Hwang, R. Greif and C. P. Grigoropoulos, "Hydrogen Production with a Solar Steam-Methanol Reformer and Colloid Nanocatalyst," International Journal of Hydrogen Energy, Vol. 341835, 2009, pp. 118-126.

[12] S. L. Smitha, K. M. Nissamudeen, D. Philip and K. G. Gopchandran, "Studies on Surface Plasmon Resonance and Photoluminescence of Silver Nanoparticles," Spectrochimica Acta Part A, Vol. 71, No. 1, 2008, pp. 186190. http://dx.doi.org/10.1016/j.saa.2007.12.002

[13] O. A. Yeshchenko, I. M. Dmitruk, A. A. Alexeenko, M. Yu. Losytskyy, A. V. Kotko and A. O. Pinchuk, "SizeDependent Surface-Plasmon-Enhanced Photoluminescence from Silver Nanoparticles Embedded in Silica," Physical Review, Vol. 79, No. 136, 2009, pp. 1-7.

[14] E. Akman, B. GencOztoprak, M. Gunes, E. Kacar and A. Demir, "Effect of Femtosecond Ti: Sapphire Laser Wavelengths on Plasmonic Behaviour and Size Evolution of Silver Nanoparticles," Photonics and NanostructuresFundamentals and Applications, Vol. 9, No. 3, 2011, pp. 276-286.

http://dx.doi.org/10.1016/j.photonics.2011.05.004

[15] A. V. Simakin, V. V. Voronov, N. A. Kirichenko and G. A. Shafeev, "Nanoparticles Produced by Laser Ablation of Solids in Liquid Environment," Applied Physics A, Vol. 791, No. 127, 2004, pp. 1127-1132.

[16] R. M. Tilaki, A. IrajiZad and S. M. Mahdavi, "Stability, Size and Optical Properties of Silver Nanoparticles Prepared by Laser Ablation in different carrier Media," Applied Physics A, Vol. 84, No. 1-2, 2006, pp. 215-219. http://dx.doi.org/10.1007/s00339-006-3604-2

[17] T. C. Prathna, N. Chandrasekaran and A. Mukherjee, "Studies on Aggregation Behaviour of Silver Nanoparticles in Aqueous Matrices: Effect of Surface Functionalization and Matrix Composition," Colloids and Surfaces A: Physicochemical and Engineering Aspects, Vol. 390, No. 1-3, 2011, pp. 216-224. http://dx.doi.org/10.1016/j.colsurfa.2011.09.047

[18] T. Tsuji, K. Iryo, Y. Nishimura and M. Tsuji, "Preparation of Metal Colloids by a Laser Ablation Technique in Solution: Influence of Laser Wavelength on the Ablation Efficiency (II)," Journal of Photochemistry and Photobiology A: Chemistry, Vol. 145, No. 3, 2001, pp. 201-207. http://dx.doi.org/10.1016/S1010-6030(01)00583-4

[19] S. Link and M. A. EL-Sayed, "Shape and Size Dependence of Radiative, Non-Radiative and Photothermal Properties of Gold Nanocrystals," International Reviews in Physical Chemistry, Vol. 19, No. 3, 2000, pp. 409-453. http://dx.doi.org/10.1080/01442350050034180

[20] H. S. Desarkar, P. Kumbhakar and A. K. Mitra, "Synthesis of Silver Hollow Nanoparticles and Observation of Photoluminescence Emission Properties," Journal of Luminescence, Vol. 134, 2013, pp. 1-7. http://dx.doi.org/10.1016/j.jlumin.2012.10.007

[21] B. Balamurugana and T. Maruyama, "Evidence of an Enhanced Interband Absorption in Au Nanoparticles: SizeDependent Electronic Structure and Optical Properties," Applied Physics Letters, Vol. 87, 2005, Article ID: 143105. http://dx.doi.org/10.1063/1.2077834

[22] M. B. Kasture, P. Patel, A. A. Prabhune, C. V. Ramana, A. A. Kulkarni and B. L. V. Prasad, "Synthesis of Silver Nanoparticles by Sophorolipids: Effect of Temperature and Sophorolipid Structure on the Size of Particles," Journal of Chemical Sciences, Vol. 120, No. 6, 2008, pp. 515-520. http://dx.doi.org/10.1007/s12039-008-0080-6

[23] M. Fayaz, C. S. Tiwary, P. T. Kalaichelvan and R. Venkatesan, "Blue Orange Light Emission from Biogenic Synthesized Silver Nanoparticles Using Trichoderma viride," Colloids and Surfaces B: Biointerfaces, Vol. 75, No. 1, 2010, pp. 175-178.

http://dx.doi.org/10.1016/j.colsurfb.2009.08.028

[24] A. Yeshchenko, M. Dmitruk, A. Alexeenko, Yu. Losytskyy, V. Kotko and O. Pinchuk, "Size-Dependent Surface-Plasmon-Enhanced Photoluminescence from Silver Nanoparticles Embedded in Silica," Physical Review B, Vol. 79, 2009, Article ID: 235438. http://dx.doi.org/10.1103/PhysRevB.79.235438

[25] P. Apell, R. Monreal and S. Lundqvist, "Photoluminescence of Noble Metals," Physica Scripta, Vol. 38, No. 2, 1988, p. 178. http://dx.doi.org/10.1088/0031-8949/38/2/012

[26] M. B. Mohamed, V. Volkov, S. Link and M. A. El-Sayed, "The 'Lightning' Gold Nanorods: Fluorescence Enhancement of over a Million Compared to the Gold Metal," Chemical Physics Letters, Vol. 317, 2000, pp. 517-523.

[27] A. P. Zhang, J. Z. Zhang and Y. Fang, "Photoluminescence from Colloidal Silver Nanoparticles," Journal of Luminescence, Vol. 128, No. 10, 2008, pp. 1635-1640. http://dx.doi.org/10.1016/j.jlumin.2008.03.014

[28] D. Basak, S. Karan and B. Mallik, "Size Selective Photoluminescence in Poly(methyl methacrylate) Thin Solid Films with Dispersed Silver Nanoparticles Synthesized by a Novel Method," Chemical Physics Letters, Vol. 420, No. 1-3, 2006, pp. 115-119. http://dx.doi.org/10.1016/j.cplett.2005.12.062 
[29] A. V. Akimov, A. Mukherjee, C. L. Yu, D. E. Chang, A. S. Zibrov, P. R. Hemmer, H. Park and M. D. Lukin, "Generation of Single Optical Plasmons in Metallic Na- nowires Coupled to Quantum Dots," Nature, London, Vol. 450, No. 402, 2007, pp. 402-406. 